\title{
Recommendations for fertility preservation in patients with lymphomas
}

\author{
Kirsten Tryde Schmidt • Claus Yding Andersen • \\ on behalf of ISFP Practice Committee
}

Received: 24 April 2012 / Accepted: 25 April 2012 / Published online: 6 May 2012

(C) Springer Science+Business Media, LLC 2012

\begin{abstract}
Purpose This guideline aims to serve as a reference for fertility specialists and other specialists working with young patients at risk of premature ovarian insufficiency (POI) or testicular dysfunction (TD) due to treatment of Hodgkin or Non-Hodgkin lymphoma.

Methods PubMed search of articles addressing risk of POI and TD according to different treatment protocols used in lymphoma patients. PubMed search of articles presenting different options for fertility treatment in cancer patients.

Results The risk of POI/TD depends on the protocol used with the highest risk in patients treated with haematopoietic stem cell transplantation/bone marrow transplantation (HSCT/BMT) and the lowest risk in patients treated with ABVD (Adriamycin, Bleomycin, Vincristine and Decarbazine). The different options of fertility preservation are discussed and their relevance according to treatment protocol, age of the patient and urgency to start treatment.

Conclusion Fertility issues should be discussed with all women of fertile age. Fertility preservation should be offered to
\end{abstract}

Capsule Fertility issues should be discussed with patients with lymphomas and fertility preservation offered when needed.

ISFP Practice Committee: S. Samuel Kim, Jacques Donnez, Pedro Barri, Antonio Pellicer, Pasquale Patrizio, Zev Rosenwaks, Peter Nagy, Tommaso Falcone, Claus Andersen, Outi Hovatta, Hamish Wallace, Dror Meirow, Debra Gook, Seok H Kim, Chii-Ruey Tzeng, Shuetu Suzuki, Bunpei Ishizuka, Marie-Madeleine Dolmans

\section{K. T. Schmidt $(\bowtie)$}

The Fertility Clinic, Rigshospitalet - University Hospital of Copenhagen,

Copenhagen, Denmark

e-mail: kirsten.tryde.schmidt@rh.regionh.dk

C. Y. Andersen

The Laboratory of Reproductive Biology,

Rigshospitalet - University Hospital of Copenhagen,

Copenhagen, Denmark young women when relevant. Children should be informed together with their parents. All men should be offered semen cryopreservation regardless of protocol used. At present, there are no established methods of fertility preservation in prepubertal boys. This guideline offers suggestions to the most preferred methods of fertility preservation according to treatment protocol, age of the patient, and urgency to start treatment.

Keywords Cancer $\cdot$ Cryopreservation $\cdot$ Fertility preservation $\cdot$ Lymphoma $\cdot$ Ovary $\cdot$ Sperm

\section{Background}

Both Hodgkin (HD) and non-Hodgkin lymphomas (NHL) are rare cancers with an incidence of 2-3/100,000 for HD and 7-12/100,000 for NHL [1]. The 5-year survival rates for both sexes and all ages combined is $85 \%$ for HD and 50 $60 \%$ for NHL [1].

\section{Treatment and risk of ovarian failure in female patients}

A number of protocols are applied in the treatment of HD dependent on stage of disease and other factors. Standard first line treatment is often ABVD (Adriamycin, Bleomycin, Vincristine and Decarbazine), which very rarely results in premature ovarian insufficiency (POI) [2-4]. Treatments following protocols that contain alkylating agents induce POI more often, varying from 20 to $85 \%$ depending on the protocol [5-7]. Patients treated with bone marrow transplantation (BMT) run a high risk of POI due to the preconditioning protocol with high dose alkylating agents and/ or total body irradiation (TBI), especially if treated as adults [6, 8-11]. Abdominal irradiation also causes POI in most cases depending on age of the patient, location of radiation field and total dose received [12] 
Treatment and risk of testicular failure in male patients

Treatment with MOPP (Nitrogen mustard, Oncovin, Procarbazine and prednisone) for HD causes azoospermia in 85$90 \%$ of patients after $>3$ courses $[13,14]$. Gonadotoxicity of the ABVD protocol is mild with $90 \%$ of patients having normal sperm counts 12 months after therapy [15]. In general, protocols containing alkylating agents and/or nitrogen mustard pose a high risk of inducing permanent impairment of spermatogenesis [16]. Treatment with BMT has a high risk of causing gonadal damage in men and boys [16]. The testes are very sensitive to irradiation and doses of $>4$ Gy cause permanent damage [16].

\section{Options for fertility preservation in females}

Co-treatment with a GnRH-analog

Randomized controlled trials using a Gonadotropin Releasing Hormone agonist (GnRH-a) during treatment in order to prevent $\mathrm{POI}$ in premenopausal women during cancer treatment have shown various results. One recent study did not find GnRH-a to have any protective effect on the ovarian function [17], while another recent study did find a reduction in the incidence of POI in the GnRH-a treated women [18]

\section{Cryopreservation of oocytes}

Oocytes can be aspirated in connection with In Vitro Fertilization (IVF) and vitrified shortly after retrieval. Lately the technique has improved significantly with survival rates after thawing approaching those seen with embryo cryopreservation [19]. Certain centres now have live birth rates using vitrified oocytes similar to those achieved with fresh oocytes [19]. However, urgency to start the chemotherapy usually only allows one treatment cycle limiting the number of oocytes available for storage.

\section{Cryopreservation of embryos}

If the woman has a partner, embryos as a result of IVF can be cryopreserved prior to chemotherapy. Thawed embryos can be transferred at a later stage when the woman has been cured of her cancer. However, urgency to start the chemotherapy usually only allows one treatment cycle limiting the number of embryos available for storage.

\section{Cryopreservation of ovarian tissue}

Ovarian tissue cryopreservation (OTC) has developed during the past 1-2 decades. One entire ovary, ovarian biopsies or semi-ovaries are cryopreserved most often using the slow-freezing protocol [20] or vitrification [21]. Autotransplantation of the cryopreserved/thawed tissue has led to return of menses and endogenous hormone production in women with treatment induced POI $[22,23]$ and to the birth of currently 20 healthy infants worldwide. All births have derived from orthotopically transplanted tissue frozen using the slow freezing protocol. Studies have found the tissue to last for 3-4 years on average per transplantation depending mainly on the woman's age at the time of freezing and the amount of tissue transplanted [24].

In vitro maturation (IVM) of oocytes

IVM is a term that covers the maturation of germinal vesicle (GV)-stage oocytes from follicles no larger than $12 \mathrm{~mm}$ from stimulated or unstimulated cycles. IVM requires a laboratory that is capable of in vitro maturing these oocytes to mature metaphase II oocytes that can then either be vitrified, or fertilized and cryopreserved as embryos. Implantation rates following transfer of in vitro matured oocytes is lower than in routine IVF cycles $<10 \%$, and also the rate of early pregnancy loss seems to be higher $[25,26]$. Nevertheless, for certain cancer patients in urgent need of fertility preservation this may be an option [27]. Another possibility is to collect immature oocytes in connection to ovarian tissue freezing in order to in vitro mature them for vitrification to optimise the fertility preservation programme [28, 29].

Risk of re-introduction of the disease

Replacement of frozen embryos generated before chemotherapy does not appear to pose a risk of re-introducing the disease.

Transplantation of cryopreserved/thawed ovarian tissue from HD or NHL patients is considered low risk in terms of causing relapse, since lymphomas rarely metastasize to the ovary. Histological evaluations of crypreserved/thawed ovarian cortical biopsies have not shown any signs of malignant cell infiltration in all but one case [30,31]. Worldwide published data on women with an original diagnosis of HD or NHL with ovarian tissue transplantation have been without reports of relapse due to the transplantation.

\section{Options for fertility preservation in males}

Cryopreservation of spermatozoa

Semen cryopreservation is a well-established method to preserve fertility in men before gonadotoxic therapy with success rates after assisted reproduction techniques using the banked semen ranging from $33 \%$ to $56 \%$ [13]. One has to bear in mind, though, that often - particularly in HD patients- the 
sperm quality may be poor due to the underlying disease itself, even before start of treatment.

In cases where the man is unable to produce a semen sample by masturbation or in case of the young pubertal boys, transrectal electro-ejaculation in general anesthesia may be used; although in this latter group experience is still scarce [32].

\section{Cryopreservation of testicular tissue}

Testicular tissue cryopreservation could be an option for young pre-pubertal boys unable to produce a semen sample, although this procedure is still experimental. The frozen/thawed tissue containing the spermatogonial stem cells could either be transplanted to the testes of the boy after he has reached adulthood or xenotransplanted to a mouse for further maturation and harvest $[14,16]$. Although this has not yet been done in humans, testicular germ cell harvest, cryopreservation and transplantation has shown to be effective in mice [33].

\section{Risk of re-introduction of the disease}

Testicular tissue removed from a patient before treatment has the potential to re-introduce the disease after the patient has been cured if transplanted. This risk is considered low in patients with HD. An alternative approach to circumvent this threat is to in vitro mature the spermatogonial stem cells, although this is not yet possible in humans.

\section{Clinical recommendations in females}

\section{When to refer?}

If in doubt about the potential impact of a given treatment on a patient's fertility, oncologists or haematologists should refer the patient to a fertility expert before chemotherapy or radiation therapy to council and advice her before treatment. Young patients less than 30 years receiving only ABVD may only need to be informed about their good prognosis by their oncologist, but women receiving protocols containing an alkylating agent or radiation therapy below the diaphragm or BMT are advised to consult a fertility expert before treatment.

Who are eligible for fertility preservation?

Patients who are treated with ABVD only have a very limited risk of POI and do not require fertility preservation in most cases.

Patients treated with combination protocols including at least one alkylating agent such as BEACOPP (Bleomycin, Etoposide, Adriamycin, Cyclophosphamide, Vincristin, Procarbazine and prednisone), CHOEP (Cyclophosphamide, hydroxydaunorubicin, vincristin, etoposide and prednisone) or ChlVPP (Chlorambucil, vinblastine, procarbazine and prednisolone) should be individually assessed and offered fertility preservation if the risk of POI is high $(>30-50 \%)$ depending on age of the patient, duration of treatment and protocol used.

Patients receiving BMT should be offered fertility preservation, because of a high risk of POI.

Patients with infra-diaphragmatic disease in whom abdominal radiation is planned should be offered fertility preservation because of a high risk of POI

Which fertility preservation method to use?

Co-treatment with a GnRHa is relatively easy and is low cost, but the protective effect is still questionable.

Cryopreservation of oocytes or embryos can be performed, but often there is not enough time for the patient to undergo an IVF cycle, because the initiation of treatment against the cancer is more urgent. Pregnancy rates per frozen embryo replacement (FER) vary between 13.6 and $23.8 \%$ based on more than 66.000 FER cycles in 26 European countries [34] and even higher in the US varying from 21 to $38.7 \%$ based on 11,858 transfers (data from SART: www.sartcorsonline.com).

OTC can often be performed on a very short notice and is thus suitable as fertility preservation in patients with an urgent need before the initiation of chemotherapy. In most lymphoma patients with a need for fertility preservation it is thus recommended as first choice. Also, in the young prepubertal girls OTC is the only option for fertility preservation. Any contraindications against an operative procedure such as disseminated disease, leuko- or trombocytopenia or other factors representing a high risk of operative complications must exclude OTC. OTC and -transplantation has a high chance of re-establishing the ovarian function in terms of endogenous hormone production and return of menstrual cycles, whereas it is still not an established method of securing fertility and thus pregnancies after transplantation, although a number of children already have been born by former HD patients following transplantation of frozen/ thawed ovarian tissue [24, 35]

How long cancer treatment can be delayed is entirely up to the treating oncologist, and fertility experts should act according to their recommendations and only perform fertility preservation if time allows it.

\section{Clinical recommendations in men}

Cryopreservation and storage of sperm is easy and noninvasive and should be offered to all men prior to a cancer treatment regardless of the risk of testicular failure. 
In cases where the patient is unable to produce a semen sample, for instance in the adolescent male, electroejaculation could be considered.

In boys, cryopreservation of testicular tissue is the only option to secure fertility, but this is still experimental and should only be offered as part of a research programme.

Why is fertility preservation important?

Psychosocial research studies have shown that cancerrelated infertility has a high impact on the quality of life of cancer survivors. Securing fertility before cancer treatment should thus have a high priority given its psychological and social significance to the individual [36, 37].

\section{Summary}

The risk of gonadal failure after treatment of HD or NHL varies depending on the protocol. ABVD has a low risk of inducing gonadal failure in both men and women, whereas treatment protocols containing alkylating agents and/or nitrogen mustard possess a higher risk. The majority of patients receiving HSCT/BMT will experience gonadal failure due to the preconditioning protocol consisting of highdose chemotherapy with alkylating agents with or without TBI.

Several options exist to preserve fertility in these patients. For men cryopreservation of sperm can be performed with good results. For young, pre-pubertal boys cryopreservation of testicular tissue is the only option available, although still experimental. For women, there are several options to secure fertility with the aim to either protect the function of the ovaries (GnRH-a co-treatment) or freezing of eggs (fertilized or un-fertilized) or ovarian tissue.

\section{Conclusions}

Fertility should be discussed with all patients at risk of gonadal failure due to treatment. Ideally, this discussion should be held by a fertility expert with special interest in fertility preservation, but oncologists, haematologists, paediatricians or other specialists treating cancer patients can also address this issue with the patient before treatment. In case of minors, the discussion should take place together with the child's parents.

Cryopreservation of sperm should be offered to all men regardless of risk of gonadal failure. It is a simple, low cost and efficient method of preserving fertility, which most patients have time to undergo before treatment. In cases where it is difficult to produce a semen sample, for instance in the adolescent male, electro-ejaculation can be offered in order to obtain an ejaculate. For pre-pubertal boys the only option to secure fertility is cryopreservation of testicular tissue, but this is still experimental.

In women, different options of fertility preservation exist and whichever is applied depends upon the woman's wish, the urgency to start treatment, the age and the marital status of the patient. If the risk of POI is very low, like in patients receiving $A B V D$, fertility preservation may not be needed. For those receiving chemotherapy with alkylationg agents, abdominal radiation or BMT fertility preservation should be considered if the general health of the patient allows it. If there is time for controlled ovarian stimulation (COS), freezing of oocytes or embryos is recommended. Cryopreservation of embryos is a well established method with good chances of giving rise to a pregnancy and can be performed in women with a partner. Vitrification of oocytes can be offered to single women and the results after thawing and replacement are now nearing those obtained with embryos. However, in HD or NHL patients urgency to start treatment often does not leave time for an IVF cycle to be completed, and for these patients cryopreservation of ovarian tissue is a valid option, since it can be performed within a few days without delaying treatment. Additionally, this method secures the re-establishment of the woman's own endogenous hormone production for a number of years after transplantation. IVM can be performed either in connection to cryopreservation of ovarian tissue or alone, but the pregnancy rates are lower than after IVF with cryopreservation of embryos or vitrification of oocytes, so other options of fertility preservation should be preferred or offered as well, if possible. Co-treatment with a GnRH-a may offer some protection to the ovary against the gonadotoxicity of chemotherapy, but randomized, controlled trials show varying results, so it should not stand alone. In young, pre-pubertal girls the only option of fertility preservation is cryopreservation of ovarian tissue, which should be offered when there is a high risk of POI, for instance in those where BMT is planned.

\section{References}

1. Storm HH, Klint $\AA$, Tryggvadottir L, Gislum M, Engholm G, Bray $\mathrm{F}$, Hakulinen T. Trends in the survival of patients diagnosed with malignant neoplasms of lymphoid, haematopoietic, and related tissue in the Nordic countries 1964-2003 followed up to the end of 2006. Acta Oncol. 2010;49:694-712.

2. Brusamolino E, Lunghi F, Orlandi E, Astori C, Passamonti F, Barate C, Pagnucco G, et al. Treatment of early-stage Hodgkin's disease with four cycles of ABVD followed by adjuvant radiotherapy: analysis of efficacy and long-term toxicity. Haematologica. 2000;85:1032-9.

3. Bonadonna G, Bonfante V, Viviani S, Di Russo A, Villani F, Valagussa P. ABVD plus subtotal nodal versus involved-field radiotherapy in early-stage Hodgkin's disease: long-term results. J Clin Oncol. 2004;22:2835-41. 
4. Decanter C, Morschhauser F, Pigny P, Lefebvre C, Gallo C, Dewailly D. Anti-Müllerian hormone follow-up in young women treated by chemotherapy for lymphoma: preliminary results. Reprod Biomed Online. 2010;20:280-5.

5. Bonadonna G, Santoro A, Viviani S, Lombardi C, Ragni G. Gonadal damage in Hodgkin's disease from cancer chemotherapeutic regimens. Arch Toxicol Suppl. 1984;7:140-5.

6. Meirow D. Ovarian injury and modern options to preserve fertility in female cancer patients treated with high dose radiochemotherapy for hemato-oncological neoplasias and other cancers. Leukaemia and Lymphoma. 1999;33:65-76.

7. Behringer K, Breuer K, Reineke T, May M, Nogova L, Klimm B, et al. Secondary amenorrhea after Hodgkin's lymphoma is influenced by age at treatment, stage of disease, chemotherapy regimen, and the use of oral contraceptives during chemotherapy: a report from the German Hodgkin's lymphoma study group. J Clin Oncol. 2005;23:7555-64.

8. Sanders JE, Buckner CD, Amos D, Levy W, Appelbaum FR, Doney K, et al. Ovarian function following marrow transplantation for aplastic anemia or leukaemia. J Clin Oncol. 1988;6:813-8.

9. Teinturier C, Hartmann O, Valteau-Couanet D, Benhamou E, Bougneres PF. Ovarian function after autologous bone marrow transplantation in childhood: high-dose busulfan is a major cause of ovarian failure. Bone Marrow Transpl. 1998;22:989-94.

10. Thibaud E, Rodriguez-Macias K, Trivin C, Esperou H, Michon J, Brauner R. Ovarian function after bone marrow transplantation during childhood. Bone Marrow Transpl. 1998;21:287-90.

11. Grigg AP, McLachlan R, Zajac J, Szer J. Reproductive status in long-term bone marrow transplant survivors receiving busulfancyclophosphamide $(120 \mathrm{mg} / \mathrm{kg})$. Bone Marrow Transpl. 2000;26:1089-95.

12. Wallace WH, Shalet SM, Crowne EC, Morris-Jones PH, Gattamaneni HR. Ovarian failure following abdominal irradiation in childhood: natural history and prognosis. Clin Oncol (Royal Coll Radiologists (Gr Br)). 1989;1:75-9.

13. Dohle GR. Male infertility in cancer patients: review of the literature. Int J Urol. 2010;17:327-31.

14. Lee SJ, Schover LR, Partridge AH, Patrizio P, Wallace WH, Hagerty K, et al. American society of clinical oncology recommendations on fertility preservation in cancer patients. J Clin Oncol. 2006;24:2917-31.

15. Tal R, Botchan A, Hauser R, Yogev L, Paz G, Yavetz H. Follow-up of sperm concentration and motility in patients with lymphoma. Hum Reprod. 2000;15:1985-8.

16. Wallace WHB, Anderson RA, Irvine DS. Fertility preservation for young patients with cancer: who is at risk and what can be offered? Lancet Oncol. 2005;6:209-18.

17. Gerber B, von Minckwitz G, Stehle H, Reimer T, Felberbaum R, Maass N, et al. Effect of luteinizing hormone-releasing hormone agonist on ovarian function after modern adjuvant breast cancer chemotherapy: the GBG 37 ZORO study. J Clin Oncol. 2011;29:2334-41.

18. Del Mastro L, Boni L, Michelotti A, Gamucci T, Olmeo N, Gori S, et al. Effect of the gonadotropin-releasing hormone analogue triptorelin on the occurrence of chemotherapy-induced early menopause in premenopausal women with breast cancer. JAMA. 2011;306:269-76.

19. Cobo A, Vajta G, Remohí J. Vitrification of human mature oocytes in clinical practise. Reprod Biomed Online. 2011;19 suppl 4:1-19.

20. Newton H, Aubard Y, Rutherford A, Sharma V, Gosden R. Low temperature storage and grafting of human ovarian tissue. Hum Reprod. 1996;11:1487-91.
21. Sheikhi M, Hultenby K, Niklasson B, Lundqvist M, Hovatta O. Clinical grade vitrification of human ovarian tissue: an ultrastructural analysis of follicles and stroma in vitrified tissue. Hum Reprod. 2011;26:594-603.

22. Donnez J, Martinez-Madrid B, Jadoul P, van Langendonckt A, Demylle DMM. Ovarian tissue cryopreservation and transplantation: a review. Hum Reprod Update. 2006;12:519-35.

23. von Wolff M, Donnez J, Hovatta O, Keros V, Maltaris T, Montag $\mathrm{M}$, et al. Cryopreservation and autotransplantation of human ovarian tissue prior to cytotoxic therapy-A technique in its infancy but already successful in fertility preservation. Eur $\mathrm{J}$ Cancer. 2009;45:1547-53.

24. Schmidt KT, Rosendahl M, Ernst E, Loft A, Nyboe Andersen A, Dueholm M, et al. Autotransplantation of cryopreserved ovarian tissue in 12 women with chemotherapy-induced premature ovarian failure: the Danish experience. Fertil Steril. 2011;95:695-701.

25. Smitz JEJ, Thompson JG, Gilchrist RB. The promise of in vitro maturation in assisted reproduction and fertility preservation. Semin Reprod Med. 2011;1:24-37.

26. Suikkari AM, Söderström-Anttila V. In-vitro maturation of eggs: is it really useful? Best Pract Res Clin Obstet Gynaecol. 2007;21:145-55.

27. Maman E, Meirow D, Brengauz M, Raanani H, Dor J, Hourvitz A. Luteal phase oocyte retrieval and in vitro maturation is an optional procedure for urgent fertility preservation. Fertil Steril. 2011;95:64-7.

28. Huang JY, Tulandi T, Holzer H, Tan SL, Chian RC. Combining ovarian tissue cryobanking with retrieval of immature oocytes followed by in vitro maturation and vitrification: an additional strategy of fertility preservation. Fertil Steril. 2008;89:567-72.

29. Fasano G, Moffa F, Dechéne J, Englert Y, Demeestere I. Vitrification of in vitro matured oocytes collected from antral follicles at the time of ovarian tissue cryopreservation. Reprod Biol Endocrin. 2011;9:150.

30. Kim SS, Radford J, Harris M, Varley J, Rutherford AJ, Lieberman $\mathrm{B}$, et al. Ovarian tissue harvested from lymphoma patients to preserve fertility may be safe for autotransplantation. Hum Reprod. 2001;16:2056-60.

31. Bittinger SE, Nazaretian SP, Gook DA, Parmar C, Harrup RA, Stern CJ. Detection of Hodgkin lymphoma within ovarian tissue. Fertil Steril. 2011;95:803.e3-6.

32. Hovav Y, Dan-Goor M, Yaffe H, Almagor M. Electroejaculation before chemotherapy in adolescents and young men with cancer. Fertil Steril. 2001;76:852-3.

33. Frederickx V, Miciels A, Goosens E, De Block G, van Steirteghem AC, Tournaye H. Recovery, survival and functional evaluation by transplantation of frozen-thawed mouse germ cells. Hum Reprod. 2004;19:948-53.

34. de Mouzon J, Goossens V, Bhattacharya S, Castilla JA, Ferraretti AP, Korsak V, et al. Assisted reproductive technology in Europe, 2006: results generated from European registers by ESHRE. Hum Reprod. 2010;25:1851-62.

35. Donnez J, Silber S, Andersen CY, Demeestere I, Piver P, Meirow D, et al. Children born after autotransplantation of cryopreserved ovarian tissue. A review of 13 live births. Ann Med. 2011;43:437-50.

36. Crawshaw MA, Sloper P. 'Swimming against the tide'-the influence of fertility matters on the transition to adulthood or survivorship following adolescent cancer. Eur J Cancer Care. 2010;19:610-20.

37. Canada AL, Schover LR. The psychosocial impact of interrupted childbearing in long-term female cancer survivors. Psychooncology. 2012;21:134-43. 